\title{
Extending the Reach of Antimicrobial Stewardship to Pediatric Patients
}

\author{
Tracy N. Zembles (D) - Natasha Nakra $\cdot$ Sarah K. Parker
}

Received: October 15, 2021 / Accepted: January 10, 2022 / Published online: January 24, 2022

(C) The Author(s) 2022

\begin{abstract}
Guidance for developing and implementing antimicrobial stewardship programs for children is lacking. This review article describes unique considerations for planning antimicrobial management of children that may impact stewardship strategies. A variety of methods and training tools are described along with metrics specific to measuring antibiotic use and outcomes in children. Handshake stewardship is specifically explained and is considered a best practice. Information on stewardship in unique settings, including the neonatal intensive care unit and outpatient settings, are included.
\end{abstract}

T. N. Zembles

Department of Enterprise Safety, Children's

Wisconsin, Milwaukee, WI, USA

\section{N. Nakra}

Department of Pediatrics, University of California Davis Health and Children's Hospital, Sacramento, CA, USA

\section{S. K. Parker}

Department of Pediatrics, Children's Hospital of Colorado, Aurora, CO, USA

T. N. Zembles $(\bowtie)$

Children's Corporate Center, 999 N 92nd Street, Mail Station 450, Milwaukee, WI 53226, USA e-mail: tzembles@chw.org
Keywords: Pediatrics; Children; Antimicrobial; Antibiotic; Stewardship; Handshake

\section{Key Summary Points}

Handshake stewardship is an effective antimicrobial stewardship strategy.

Metrics to determine success need to be tailored for children.

Pediatric training tools are available and recommended.

The Core Elements can be used to develop a framework for antimicrobial stewardship.

A wide range of stakeholders are required for success.

\section{INTRODUCTION}

Antimicrobial stewardship programs (ASPs) aim to delay the propagation of antimicrobial resistance by ensuring antimicrobials are prescribed only when necessary and administered for an appropriate duration, at an appropriate dose, and via the appropriate route [1]. There are unique considerations when planning 
antimicrobial management of children that may impact ASP strategies. First, a child's body composition and organ function change over time [2]. These changes are discordant in the first decade of life, and may result in variable pharmacokinetic and pharmacodynamic outcomes associated with different antibiotic classes. Second, children are not often included in dose-finding studies [3]. Consequently, doses for children are frequently extrapolated from the adult dose, which does not account for changes in organ function over time. Third, the optimal duration of antimicrobial therapy for children is often unknown. While multiple studies exist evaluating durations of therapy for adults, there is a paucity of this same information in children [4]. Fourth, children's diets, habits, and schedules present unique challenges; for example, fluoroquinolones are chelated by milk and formula, peripherally inserted catheters are harder to protect from inadvertent removal, and overwhelmed schools are not always reliable partners in antibiotic administration. Finally, the use of antimicrobials in infants and young children may have long-term consequences through effects on the gut microbiome, such as increasing the risk of autoimmune and atopic disease [5].
Additionally, antimicrobial resistance is a growing threat in children [6]. Many of the same risk factors for resistance in adults occur in children, including chronic medical conditions, invasive medical devices, prolonged hospitalizations, use of immunosuppression, and prior antibiotic use. Healthcare for children with complex medical conditions is improving; this in turn increases the number of children with the aforementioned risk factors. Unfortunately, most large national surveillance reports do not include children's hospitals and do not report pediatric specific data related to antibiotic resistance.

In 2014, the Centers for Disease Control (CDC) recommended that all hospitals implement ASPs and published the "Core Elements of Hospital Antibiotic Stewardship Programs", which outlined the important features to promote the success of an ASP (Table 1) [7]. The Joint Commission (TJC) subsequently implemented an antimicrobial stewardship standard for acute care hospitals in January 2017, with requirements that mirrored the CDC Core Elements [8]. Essential to the implementation of an inpatient stewardship program is the selection of both a physician and pharmacy leader with expertise in infectious diseases, who work closely with administration, the microbiology

Table 1 Summary of the Centers for Disease Control and Prevention Core Elements of Hospital Antibiotic Stewardship Programs [7]

\begin{tabular}{|c|c|}
\hline Core element & Description \\
\hline $\begin{array}{l}\text { Hospital } \\
\text { leadership }\end{array}$ & Dedicate necessary human, financial, and information technology resources \\
\hline Accountability & Appoint a leader or co-leaders responsible for program management and outcomes \\
\hline $\begin{array}{l}\text { Pharmacy } \\
\text { expertise }\end{array}$ & $\begin{array}{l}\text { Appoint a pharmacist, ideally as the co-leader of the stewardship program, to lead implementation efforts } \\
\text { to improve antibiotic use }\end{array}$ \\
\hline Action & Implement interventions to improve antibiotic use \\
\hline Tracking & Monitor antibiotic prescribing, impact of interventions, and other important outcomes \\
\hline Reporting & $\begin{array}{l}\text { Regularly report information on antibiotic use and resistance to prescribers, pharmacists, nurses, and } \\
\text { hospital leadership }\end{array}$ \\
\hline Education & $\begin{array}{l}\text { Educate prescribers, pharmacists, and nurses about adverse reactions from antibiotics, antibiotic } \\
\text { resistance, and optimal prescribing }\end{array}$ \\
\hline
\end{tabular}


laboratory, infection prevention, inpatient providers (such as hospitalists), nursing, and quality improvement departments to develop and support strategies to improve antimicrobial use [9]. Following publication of the acute care requirements, the CDC published the "Core Elements of Outpatient Antibiotic Stewardship" to provide a framework for improving antibiotic prescribing in the ambulatory setting (Table 2) [10]. As expected, TJC then developed standards for antimicrobial stewardship in ambulatory healthcare based on these Core Elements which went into effect January 1, 2020 [11]. Interestingly, neither TJC nor the CDC specifies that providers need pediatric expertise to implement a pediatric ASP, nor do they specify that programs must include pediatric patients in their reviews. This unfortunately results in pediatric patients being left out of many ASPs.

The objective of this article is to review common antimicrobial stewardship strategies that are appropriate for pediatric patients. Adult and pediatric publications on antimicrobial stewardship were reviewed by TZ and NN. Personal experiences with pediatric stewardship from all three authors were included in the article. This article is based on previously conducted studies and does not contain any new studies with human participants or animals performed by any of the authors.

\section{ANTIMICROBIAL STEWARDSHIP METHODS}

There are multiple acceptable approaches to implement ASPs in children. Provider education is an attractive strategy to improve antimicrobial prescribing; however, traditional education alone does not appear to be an effective approach and must be combined with other strategies to be successful in reducing inappropriate use [12]. Restrictive approaches (e.g., preauthorization) generally consist of a phone call to the stewardship team for select antimicrobials to gain approval before the agent is dispensed by the pharmacy. This method is especially effective in the setting of an outbreak of a resistant organism or in times of drug shortages. However, disadvantages to this approach include a potential delay in administration of restricted antimicrobials as well as disruptions in workflow. Another strategy, often complementary to other methods, is the development of guidelines. Antimicrobial guidelines which focus on a specific disease state, syndrome, or procedure (e.g., community-acquired pneumonia, fever and neutropenia, and perioperative prophylaxis) should be developed by ASP leaders with input from other stakeholders in order to achieve mutually agreeable guidelines [13]. Pediatricians and pharmacists with training in infectious diseases are essential participants in the development of guidelines, especially since most national guidelines focus on adult care. Local guidelines should be

Table 2 Summary of the Centers for Disease Control and Prevention Core Elements of Outpatient Antibiotic Stewardship [10]

\begin{tabular}{ll}
\hline Core element & Description \\
\hline $\begin{array}{l}\text { Commitment } \\
\text { Action }\end{array}$ & $\begin{array}{l}\text { Demonstrate dedication to and accountability for optimizing antibiotic prescribing and patient safety } \\
\text { Implement at least one policy or practice to improve antibiotic prescribing, assess whether it is } \\
\text { working, and modify as needed }\end{array}$ \\
$\begin{array}{l}\text { Tracking and } \\
\text { reporting }\end{array}$ & $\begin{array}{l}\text { Monitor antibiotic prescribing practices and offer regular feedback to clinicians, or have clinicians } \\
\text { aducation and } \\
\text { expertise }\end{array}$ \\
\hline
\end{tabular}


available to providers in an easily accessible location, and should be reviewed regularly to reflect changes in practice and local microbiologic trends.

Prospective audit and feedback (PAF) is a more widely implemented strategy in which providers prescribe antimicrobials as they deem appropriate, but are then subsequently reviewed by the ASP team $48-72 \mathrm{~h}$ after initiation, with feedback provided as to the appropriateness of the antimicrobial choice. Traditionally, this feedback is given remotely (via the electronic medical record, paging, or e-mail), and usually only involves review of particular targeted antimicrobials. An extension of PAF now considered a best practice by the TJC is "handshake stewardship" [14]. This model was first published at the Children's Hospital of Colorado and has subsequently been adopted by several other centers around the country. In this model, the ASP team reviews all antimicrobials and performs daily in-person rounding with each of the prescribing teams to provide feedback regarding antimicrobial use. Handshake stewardship is demonstrated to be highly effective and sustainable, decreasing overall antibiotic days of therapy per 1000 patient days by $23 \%$ over 5 years in a published interrupted time series [15]. Pre-authorization and remote prospective audit and feedback, while demonstrated to decrease use of select antimicrobials in targeted populations, are not demonstrated to decrease overall use in a whole hospital to the same degree. Success of handshake stewardship is attributed to the face-to-face rounding that promotes discussion and patient-specific education about appropriate antimicrobial use, likely leading to change in the institutional culture [15]. Although implementation of a handshake stewardship model may be perceived as time- and labor-intensive, cost analyses demonstrate that the initial investment will result in substantial cost savings in antimicrobial use (2 million US dollars per year), in addition to improving quality of patient care $[16,17]$. Hospital ASPs with limited resources may consider a partial handshake approach, with in-person rounds performed in specific units or on certain days of the week. In the authors' personal experiences (TZ, NN, SP), we observe that the face-to-face time investment demonstrates to other healthcare providers that we are invested and engaged in improving quality of care, which increases the likelihood that those providers will subsequently accept a recommended intervention [18]. A summary of the advantages and disadvantages of each of these methods is described in Table 3.

In addition to the official ASP team, de-centralized pharmacists frequently play a vital role in ASP activities beginning with review of all antimicrobial orders [19]. A great tool to aid front-line pharmacists is requiring providers to specify an indication when placing an antimicrobial order; this allows the pharmacist to evaluate appropriateness for the particular clinical syndrome. Pharmacists ensure that optimal antimicrobial dosing and route of administration are selected to achieve a therapeutic concentration at the suspected site of infection. Given the limited dosing data in children, this is particularly important in pediatric stewardship. Facility-specific, pharmacydriven guidelines (including dosing recommendations) assist in improving therapy for young children. In the setting of a reported antibiotic allergy, pharmacists assist with selection of alternative regimens and may also participate in allergy reconciliation and/or delabeling when appropriate. Additionally, pharmacists frequently provide guidance regarding monitoring for adverse drug effects as well as performing therapeutic drug monitoring. They provide real-time feedback for adjustments of antimicrobial dosing or selection. In non-freestanding children's hospitals, hospital pharmacists fill a key role in pediatric ASPs which often are limited in resources and may not be supported by a pharmacist with infectious diseases training $[19,20]$.

An often unrecognized participant in the antimicrobial stewardship team is the bedside nurse. Staff nurses already perform numerous functions that support the process of antimicrobial stewardship, including taking allergy histories, monitoring and reporting adverse events, performing patient/family education, and communicating with all members of the healthcare team $[21,22]$. Professional organizations, including the American Nursing 
Table 3 Advantages and disadvantages of various antimicrobial stewardship methods

\begin{tabular}{|c|c|c|}
\hline Method & Advantages & Disadvantages \\
\hline Provider education & May influence prescribing behavior & $\begin{array}{l}\text { Must be repeated with new hires } \\
\text { Best when combined with active } \\
\text { intervention } \\
\text { Does not clearly improve } \\
\text { prescribing } \\
\text { Best educational methodology not } \\
\text { clear }\end{array}$ \\
\hline Antibiotic restrictions & $\begin{array}{l}\text { May be effective at decreasing use of targeted agents } \\
\text { Results in cost savings }\end{array}$ & $\begin{array}{l}\text { May increase staffing requirements } \\
\text { May delay therapy } \\
\text { May increase use of alternative } \\
\text { agents } \\
\text { May not decrease overall use } \\
\text { Perceived loss of prescriber } \\
\text { autonomy } \\
\text { Stewards seen as "police" }\end{array}$ \\
\hline Development of guidelines & $\begin{array}{l}\text { Improves prescribing behavior (drug selection, dosing, } \\
\text { duration) } \\
\text { Reduces practice variations }\end{array}$ & $\begin{array}{l}\text { Requires adherence } \\
\text { Does not necessarily decrease } \\
\text { overall use } \\
\text { Time consuming to develop and } \\
\text { measure }\end{array}$ \\
\hline $\begin{array}{l}\text { Prospective audit and } \\
\text { feedback (PAF) }\end{array}$ & $\begin{array}{l}\text { May be effective at decreasing use of targeted agents } \\
\text { Avoids delays in initiation of therapy } \\
\text { Maintains prescriber autonomy }\end{array}$ & $\begin{array}{l}\text { May increase staffing requirements } \\
\text { May be difficult to reach providers }\end{array}$ \\
\hline Handshake stewardship & $\begin{array}{l}\text { May be effective at decreasing overall use } \\
\text { Promotes acceptance } \\
\text { Allows for education in combination with prospective } \\
\text { audit and feedback } \\
\text { Promotes collaboration } \\
\text { May decrease diagnostic error }\end{array}$ & May increase staffing requirements \\
\hline
\end{tabular}

Association and the CDC support the incorporation of nurses in antimicrobial stewardship [23]. This is especially important in pediatrics because nurses best understand the impact of antimicrobial use on the day-to-day lives of hospitalized children, where minimizing discomfort and maintaining schedules for feeding and sleep are so important [24]. For example, administering an oral antibiotic to a 3-year-old is not always easy, repeated blood draws create 
distrust, and "simple" antibiotic adverse drug events, like diaper rash, impact patient and family satisfaction. Furthermore, nurses should be encouraged to participate in daily stewardship; one key intervention is to question the reason for use, choice, route, and duration of antimicrobials. Nurses can provide input regarding readiness for enteral therapy, provide real-time information regarding culture results, and help educate patients and families regarding antibiotic use.

Overall, we observe that the handshake stewardship approach, in collaboration with decentral pharmacists and bedside nurses, works well in pediatric stewardship. Perhaps this is because pediatric care, by necessity, is already based on a team approach. Parents are usually involved in the care and daily decision-making of the child, pharmacists are essential in evaluating appropriateness of ordered medications, and nurses often serve as advocates for children, especially in the absence of parental presence.

\section{STEWARDSHIP IN UNIQUE SETTINGS}

The neonatal intensive care unit (NICU) poses a unique challenge for pediatric ASPs. First, dosing varies based on gestational and post-menstrual age, and pharmacokinetic studies of many antimicrobials are lacking in the premature neonatal population. Next, diagnosis of serious bacterial infection in neonates is difficult, due to non-specific clinical manifestations of sepsis, inability to rely on biomarkers of sepsis such as C-reactive protein, and small blood volumes typically submitted for blood culture which decrease sensitivity [25]. Neonatal providers are often reluctant to stop antibiotics because missing a serious bacterial infection in a neonate can be devastating and impact future development. Some effective strategies specific to the NICU setting include limiting empiric antibiotic use for culture-negative sepsis or pneumonia, using neonatal sepsis calculators to inform when to start antibiotics, and developing empiric guidelines for sepsis and necrotizing enterocolitis based on NICUspecific antibiograms and with input from neonatologists and other specialists [26, 27]. Other unique inpatient settings include pediatric hospitals within an adult hospital, where resources and electronic medical records (EMR) are often geared to adult patients and hospitals in resource-limited settings, where challenges may include minimal infection control, high levels of antibiotic resistance, limited drug supply, inadequate access to microbiology, and lack of EMRs.

Though antimicrobial stewardship standards were initially implemented within the acute care setting, it may be more important to address antimicrobial use in the outpatient setting given the overall burden of use. Proportionally, antibiotic consumption is largely driven by use in young children with approximately one-quarter of all pediatric ambulatory visits resulting in an antibiotic prescription [28]. For pediatric programs with only an inpatient stewardship team, one way to ease into the outpatient environment is to evaluate prescriptions for children discharged from the hospital with an antibiotic to complete at home. Olson et al. evaluated a random sample of 150 antibiotic prescriptions for children being discharged from the hospital [29]. Of those, nearly one in three $(27 \%)$ were suboptimal in some way, with duration of therapy reported as the most frequent reason for suboptimal prescribing. This transitional setting also provides an opportunity to teach outpatient stewardship principles to inpatient learners.

Although starting with discharge prescriptions is reasonable, programs must find ways to evaluate and improve antibiotic use in nonhospital settings as well. Hersh et al. evaluated antibiotic use among children in ambulatory care [30]. Respiratory conditions accounted for more than $70 \%$ of visits where antibiotics were prescribed. Of these, approximately one-quarter were for respiratory conditions where antibiotics were not clearly indicated. In the outpatient setting, provider feedback as to appropriateness of antimicrobial prescribing with peer comparison can be used to improve antibiotic use in ambulatory care. Gerber et al. implemented this method among 18 ambulatory practices, comprising 170 clinicians [31]. 
The program focused on antibiotic choice for acute respiratory tract infections with established guidelines (acute sinusitis, streptococcal pharyngitis, and pneumonia) for which narrowspectrum antibiotics are recommended. As a result, broad spectrum use across all 18 sites declined by $12.5 \%$. Unfortunately, prescribing rates returned to pre-intervention rates when the process was terminated, supporting the need for ongoing stewardship initiatives.

\section{ANTIMICROBIAL STEWARDSHIP METRICS}

ASPs should periodically perform analyses to determine whether they are effective in improving antimicrobial utilization and adherence to facility-specific guidelines. Standardized measures of antimicrobial use are typically utilized to evaluate the success of an ASP, with the caveat that they do not necessarily reflect changes in antimicrobial resistance. Though defined daily doses (DDD) are typically used in adult settings, days of therapy per 1000 patientdays (DOT) is a more commonly utilized process measure in children. DOT is defined as the number of unique antibiotics received per hospital day. This metric is preferred in the pediatric setting as it does not rely on antibiotic dose (used in DDD calculation), which is highly dependent on patient age and weight. DOT is a standard measure and can therefore be used for inter-facility comparison. All US hospitals are encouraged to voluntarily submit antimicrobial utilization data (in the form of DOT) to the National Healthcare Safety Network (NHSN) Antimicrobial Use and Resistance (AUR) module, which provides risk-adjusted benchmarking against other facilities with similar patient populations [32]. The AUR module provides benchmarking for specific pediatric and neonatal units and specific categories of antimicrobial agents used in the pediatric and neonatal settings. Wattier et al. described another method to adjust antimicrobial use measurements on the basis of case mix at individual children's hospitals to account for variability in antimicrobial use and subsequently allowed for more appropriate inter-facility comparisons [33]. Results of internal and external ASP metrics should be shared periodically with prescribing providers and hospital administrators to demonstrate ASP successes and identify areas for improvement.

Measuring antibiotic use in DOT has limitations as a metric, however. For example, broadspectrum monotherapy (such as a carbapenem) would result in a lower DOT than a more narrow-spectrum combination therapy (such as a third-generation cephalosporin plus metronidazole). In order to circumvent this, novel approaches to assess the spectrum of antimicrobial therapy were developed, in which a "spectrum score" is assigned on the basis of the number or types of pathogens targeted by a specific regimen [34]. ASPs can utilize this score to assess whether a new guideline for a specific clinical syndrome is effective in decreasing broad-spectrum use, or whether stewardship interventions result in de-escalation from an initial broad-spectrum empiric regimen to a narrower focused regimen. Other metrics in development include those that assess antibiotic "appropriateness", although the subjectivity of these measures makes it difficult to compare across settings or health systems. Trivedi et al. recently reported on the use of a standardized intensive care unit antibiotic point prevalence tool to assess appropriateness that was implemented across 12 hospitals and successfully allowed for benchmarking across hospitals [35].

\section{ANTIMICROBIAL STEWARDSHIP TRAINING TOOLS}

Multiple ASP training programs exist for practitioners who are currently or planning to pursue stewardship activities. These include Making a Difference in Infectious Diseases ASP certificate, the Society of Infectious Diseases Pharmacists ASP Certificate Program, CDC Training on Antimicrobial Stewardship, and The Society for Healthcare Epidemiology of America Antibiotic Stewardship Training Course. All of these programs are designed to educate pharmacists, physicians, and other ASP team members regarding fundamental elements 
of implementing, managing, and improving ASP programs. However, none of these focus on pediatrics. The Pediatric Infectious Diseases Society, therefore, developed a "Pediatric ASP Toolkit" (available at https://pids.org/pediatricasp-toolkit/) to provide helpful resources for improving the use of antibiotics in children cared for in all pediatric healthcare settings. This toolkit includes a "how-to guide" for both inpatient and outpatient stewardship programs. An additional resource for pediatric outpatient stewardship is the Dialogue Around Respiratory Illness Treatment (DART) module (available at https://www.uwimtr.org/dart/), which addresses the largest driver of outpatient prescribing for children: the prescriber's perception of what parents want. The module teaches providers how to address prescribing in an effective, timeefficient manner.

\section{CONCLUSION}

Antimicrobial stewardship methods directed at improving healthcare for children include prospective audit with feedback, specifically, "handshake stewardship", as an effective strategy. Metrics to determine success need to be tailored for children. Tools specific to the pediatric population are available and recommended for institutions looking to establish stewardship programs. The Core Elements can be used to help teams develop a framework for antimicrobial stewardship. A wide range of stakeholders are required to ensure success.

\section{ACKNOWLEDGEMENTS}

The authors would like to thank Katie Ray for proofreading the manuscript.

Funding. No funding or sponsorship was received for this study or publication of this article.

Authorship. All named authors meet the International Committee of Medical Journal Editors (ICMJE) criteria for authorship for this article, take responsibility for the integrity of the work as a whole, and have given their approval for this version to be published.

Author Contributions. All authors contributed to the manuscript conception. The first draft was written by Tracy Zembles and all authors commented on previous versions of the manuscript. All authors read and approved the final manuscript.

Disclosures. Tracy N Zembles, Natasha Nakra and Sarah K Parker have nothing to disclose.

Compliance with Ethics Guidelines. This article is based on previously conducted studies and does not contain any new studies with human participants or animals performed by any of the authors.

Open Access. This article is licensed under a Creative Commons Attribution-NonCommercial 4.0 International License, which permits any non-commercial use, sharing, adaptation, distribution and reproduction in any medium or format, as long as you give appropriate credit to the original author(s) and the source, provide a link to the Creative Commons licence, and indicate if changes were made. The images or other third party material in this article are included in the article's Creative Commons licence, unless indicated otherwise in a credit line to the material. If material is not included in the article's Creative Commons licence and your intended use is not permitted by statutory regulation or exceeds the permitted use, you will need to obtain permission directly from the copyright holder. To view a copy of this licence, visit http://creativecommons.org/licenses/by$\mathrm{nc} / 4.0 /$.

\section{REFERENCES}

1. Fishman N. Policy statement on antimicrobial stewardship by the Society for Healthcare Epidemiology of America (SHEA), the Infectious Diseases Society of America (IDSA), and the Pediatric Infectious Diseases Society (PIDS). Infect Control Hosp Epidemiol. 2012;33(4):322-7. 
2. Weber DR, Leonard MB, Zemel BS. Body composition analysis in the pediatric population. Pediatr Endocrinol Rev. 2012;10(1):130-9.

3. Korth-Bradley JM. The path to perfect pediatric posology - drug development in pediatrics. J Clin Pharmacol. 2018;58(Supp 10):S48-57.

4. Spellberg B. Duration of antibiotic therapy: shorter is better. Ann Intern Med. 2019;171(3):210-1.

5. Neuman H, Forsythe P, Uzan A, Avni O, Koren O. Antibiotics in early life: dysbiosis and the damage done. FEMS Microbiol Rev. 2018;42(4):489-99.

6. Medernach RL, Logan LK. The growing threat of antibiotic resistance in children. Infect Dis Clin N Am. 2018;32(1):1-17.

7. Centers for Disease Control and Prevention. Core elements of hospital antibiotic stewardship programs. 2021. https://www.cdc.gov/antibiotic-use/ core-elements/hospital.html. Accessed 25 Aug 2021.

8. The Joint Commission. Approved: new antimicrobial stewardship standard. 2021. https://www. jointcommission.org/-/media/enterprise/tjc/impor ted-resource-assets/documents/new_antimicrobial stewardship_standardpdf.pdf?db=web\&hash $=6930$ 7456CCE435B134854392C7FA7D76\&hash $=69307$ 456CCE435B134854392C7FA7D76. Accessed 25 Aug 2021.

9. Heil E, Kuti J, Bearden D, Gallagher J. The essential role of pharmacists in antimicrobial stewardship. Infect Control Hosp Epidemiol. 2016;37(7):753-4.

10. Sanchez GV, Fleming-Dutra KE, Roberts RM, Hicks LA. Core elements of outpatient antibiotic stewardship. MMWR Recomm Rep. 2016;65(6):1-12.

11. The Joint Commission. Antimicrobial stewardship in ambulatory health care. R3 Report, Issue 23. 2019. https://www.jointcommission.org/-/media/ tjc/documents/standards/r3-reports/r3_23_ antimicrobial_stewardship_amb_6_14_19_final2. pdf. Accessed 30 June 2019.

12. Barlam TF, Cosgrove SE, Abbo LM, et al. Implementing an antibiotic stewardship program: guidelines by the Infectious Diseases Society of America and the Society for Healthcare Epidemiology of America. Clin Infect Dis. 2016;62(10):e51-77.

13. Lee KR, Bagga B, Arnold SR. Reduction of broadspectrum antimicrobial use in a tertiary children's hospital post antimicrobial stewardship program guideline implementation. Pediatr Crit Care Med. 2016;17(3):187-93.
14. Hurst AL, Child J, Pearce K, Palmer C, Todd JK, Parker SK. Handshake stewardship: a highly effective rounding-based antimicrobial optimization service. Pediatr Infect Dis J. 2016;35(10):1104-10.

15. MacBrayne CE, Williams MC, Levek C, et al. Sustainability of handshake stewardship: extending a hand is effective years later. Clin Infect Dis. 2020;70(11):2325-32.

16. Parker SK, Hurst AL, Thurm C, et al. Anti-infective acquisition costs for a stewardship program: getting to the bottom line. Clin Infect Dis. 2017;65(10): 1632-7.

17. Searns JB, Williams MC, MacBrayne CE, et al. Handshake antimicrobial stewardship as a model to recognize and prevent diagnostic errors. Diagnosis. 2020;8(3):347-52.

18. Hurst AL, Child J, Parker SK. Intervention and acceptance rates support handshake stewardship strategy. J Pediatric Infect Dis Soc. 2019;8(2):162-5.

19. Nguyen-Ha PT, Howrie D, Crowley K, et al. A quality assessment of a collaborative model of a pediatric antimicrobial stewardship program. Pediatrics. 2016;137(5): e20150316.

20. Turner RB, Valcarlos E, Loeffler AM, Gilbert M, Chan D. Impact of an antimicrobial stewardship program on antibiotic use at a non-freestanding children's hospital. J Pediatric Infect Dis Soc. 2017;6(3):e36-40.

21. Olans RN, Olans RD, DeMaria A Jr. The critical role of the staff nurse in antimicrobial stewardship unrecognized, but already there. Clin Infect Dis. 2016;62(1):84-9.

22. Monsees EA, Tamma PD, Cosgrove SE, Miller MA, Fabre V. Integrating bedside nurses into antibiotic stewardship: a practical approach. Infect Control Hosp Epidemiol. 2019;40(5):579-84.

23. American Nurses Association, CDC. Redefining the antibiotic stewardship team: recommendations from the American Nurses Association/Centers for Disease Control and Prevention Workgroup on the role of registered nurses in hospital antibiotic stewardship practices. JAC Antimicrob Resist. 2019;1(2):dlz037.

24. Mozer CL, Bhagat PH, Seward SA, et al. Optimizing oral medication schedules for inpatient sleep: a quality improvement intervention. Hosp Pediatr. 2021;11(4):327-33.

25. Cantey JB, Patel SJ. Antimicrobial stewardship in the NICU. Infect Dis Clin N Am. 2014;28(2): 247-61. 
26. Kuzniewicz MW, Puopolo KM, Fischer A, et al. A quantitative, risk-based approach to the management of neonatal early-onset sepsis. JAMA Pediatr. 2017;171(4):365-71.

27. Cotten CM, McDonald S, Stoll B, Goldberg RN, Poole K, Benjamin DK Jr. National Institute for Child Health and Human Development Neonatal Research Network. The association of third-generation cephalosporin use and invasive candidiasis in extremely low birth-weight infants. Pediatrics. 2006;118(2):717-22.

28. Hicks LA, Bartoces MG, Roberts RM, et al. US outpatient antibiotic prescribing variation according to geography, patient population, and provider specialty in 2011. Clin Infect Dis. 2015;60(9):1308-16.

29. Olson J, Thorell E, Hersh A. Evaluation of discharge antibiotic prescribing at a freestanding children's hospital: opportunities for stewardship. J Pediatr Infect Dis Soc. 2019;8(6):563-6.

30. Hersh AL, Shapiro DJ, Pavia AT, Shah SS. Antibiotic prescribing in ambulatory pediatrics in the United States. Pediatrics. 2011;128(6):1053-61.

31. Gerber JS, Prasad PA, Fiks AG, et al. Effect of an outpatient antimicrobial stewardship intervention on broad-spectrum antibiotic prescribing by primary care pediatricians: a randomized trial. JAMA. 2013;309(22):2345-52.

32. Centers for Disease Control and Prevention, National Healthcare Safety Network (NHSN). Antimicrobial use and resistance module. 2021. https://www.cdc.gov/nhsn/psc/aur/index.html. Accessed 25 Aug 2021.

33. Wattier RL, Thurm CW, Parker SK, Banerjee R, Hersh AL. Pediatric health information system antimicrobial stewardship research group. Clin Infect Dis. 2021;73(5):925-32.

34. Poole NM, Wattles BA, El Feghaly RE. Proposed metrics to benchmarck antibiotic prescribing in pediatric outpatient settings. Am J Infect Control. 2021;49(12):1547-50.

35. Trivedi KK, Bartash $\mathrm{R}$, Letourneau $\mathrm{AR}$, et al. Opportunities to improve antibiotic appropriateness in US ICUs: a multicenter evaluation. Crit Care Med. 2020;48(7):968-76.

\section{Publisher's Note}

Springer Nature remains neutral with regard to jurisdictional claims in published maps and institutional affiliations. 